\title{
Téléphoner au volant : impact sur la conduite et risque d'accident une revue de la littérature
}

\section{Phoning while driving: impact on the driving and risk of accident a literature review}

\author{
Marie-Pierre Bruyas $^{1}$ et Jean-Louis Martin ${ }^{2 \& 3}$ \\ IFSTTAR-LESCOT $^{1-}$ IFSTTAR-UMRESTTE ${ }^{2}$ \\ 25, avenue François Mitterrand, F-69675 Bron, France \\ Université de Lyon ${ }^{3}$, F-69622 Lyon, France \\ marie-pierre.bruyas@ifsttar.fr - Tel : +33 472142459 \\ Jean-louis.martin@ifsttar.fr - Tel : +33 472142512 \\ Correspondant principal : Marie-Pierre Bruyas
}

Résumé : Ce papier propose une revue de la littérature issue des études expérimentales, épidémiologiques et naturalistes ayant tenté de mesurer d'une part, l'impact de l'usage du téléphone sur la conduite automobile et, d'autre part, le risque d'accident associé à cette pratique. La combinaison de ces différentes études a permis tout d'abord de montrer que la tâche de conduite était bien altérée lorsque l'on utilise son téléphone au volant et de quantifier cette perturbation (études expérimentales) puis de vérifier que cette perturbation était bien susceptible d'être à l'origine d'accidents et de quantifier le risque encouru (études épidémiologiques). Les études naturalistes de suivi de flottes de véhicules, tout en apportant certaines précisions, tendent à confirmer ces résultats. Avantages et limites de chaque approche sont également discutés.

\begin{abstract}
This paper gives a literature review from experimental, epidemiological and naturalistic studies, having tried to measure on the one hand, the impact of mobile phone use on the driving and, on the other hand, the risk of accident associated with this practice. The overview of these studies allowed first of all to show that the driving task is altered during the period of mobile phone use and to quantify the disturbance (experimental studies) then to verify that this disturbance can be at the origin of accidents and quantify this risk (epidemiological studies). The naturalist studies consisting in following-up fleet vehicles, give some more explanations and tend to confirm the results. Advantages and limits of each approach are also discussed.
\end{abstract}


Mots clés : Téléphone ; Conduite automobile ; Attention ; Distraction ; Risque d'accident, Etudes expérimentales, Etudes épidémiologiques, études naturalistes

Keywords: Mobile phone; Driving; Attention; Distraction; Accident; Experimental studies; epidemiology; naturalistic studies

\section{Introduction}

Avec le développement des systèmes d'information et de communication embarqués, les sources d'informations données au conducteur se multiplient. Si cette évolution offre certaines perspectives d'amélioration de la sécurité et du confort de la conduite, elle soulève également des questionnements en ce qui concerne les risques de distraction et de modifications du comportement de conduite qui peuvent être engendrés. En effet, l'usage de ces systèmes génère des situations de tâches ajoutées à la tâche de conduite, qui ne sont pas toujours sans conséquence en termes de sécurité routière.

De très nombreuses recherches se sont focalisées sur l'effet du téléphone sur la conduite automobile. Ce papier se propose d'en faire une synthèse en les différenciant selon trois grands types : les études expérimentales qui ont observé le comportement d'un conducteur en train de téléphoner pour déterminer l'impact de cette activité sur la conduite, les études épidémiologiques qui visent à évaluer le risque d'accident associé à cette utilisation et, enfin, les études naturalistes, plus récentes, qui ont tenté de préciser ce risque en fonction des actions réalisées par le conducteur lorsqu'il téléphone. Chaque type d'études donne un éclairage différent sur la question.

\section{1. Études expérimentales}

Elles ont été réalisées dans différents contextes : en laboratoire, sur piste d'essais ou sur route réelle. Différents indicateurs du comportement sont pris en compte et comparés lorsque le conducteur téléphone et lorsqu'il ne téléphone pas. La plupart d'entre elles se sont focalisées sur l'effet d'une conversation téléphonique. Certaines ont également mesuré les perturbations enregistrées lorsque le conducteur manipule son téléphone (numérotation, prise de ligne, envoi ou rédaction de SMS...).

\subsection{Effet d'une conversation téléphonique sur la prise et le traitement de l'information routière}

De nombreux travaux ont montré l'importance des processus de prélèvement et de traitement de l'information en conduite. En effet, regarder dans la mauvaise direction à un moment critique et/ou ne pas voir un élément important de l'environnement routier peuvent avoir des conséquences dramatiques lorsque l'on conduit.

\subsubsection{Prélèvement de l'information}

Des modifications importantes du comportement visuel sont observées chez les conducteurs lorsqu'ils conversent au téléphone au volant. Il a été ainsi montré qu'ils regardent davantage droit devant et négligent la consultation de leur champ périphérique, notamment des rétroviseurs et des organes de contrôle tels que le compteur de vitesse [1-5]. Parallèlement, une réduction de la variabilité de la direction spatiale des regards (ou concentration des regards) vers la zone centrale de la route a pu être mise en évidence, lorsque les conducteurs réalisent des tâches cognitives, telles que discuter au téléphone [2][4][6-9]. Ce phénomène de 
concentration des regards associé à une moindre consultation du champ périphérique est révélateur d'une altération des stratégies de prise d'information visuelle et pourrait révéler, ainsi que le suggèrent Victor et coll. [8], que lorsqu'il téléphone, un conducteur accorde la priorité au contrôle de sa trajectoire au détriment d'autres sous-tâches de la conduite.

Par ailleurs, Recarte et Nunes [6] ont montré que la concentration des regards engendrée par la pratique d'une activité mentale au volant s'accompagne d'une diminution significative de la capacité à détecter des cibles. Toutefois pour ces auteurs, cette altération de la perception ne serait pas nécessairement une conséquence de la concentration des regards mentionnée plus haut, mais serait plutôt liée au détournement de ressources attentionnelles vers la tâche distractive au détriment de la tâche de conduite, ce qui nuirait à une mise en jeu optimale des processus cognitifs. Cette détérioration des performances de détection est également montrée dans d'autres études [10-13].

\subsubsection{Traitement de l'information}

Les travaux de Strayer et de ses collaborateurs [14-16] ont cherché à expliquer les déficits perceptifs enregistrés lorsqu'un conducteur effectue une activité cognitive. Ils ont proposé comme cadre explicatif l'hypothèse d'une cécité inattentionnelle ( inattentional blindness ») engendrée par l'action de converser en conduisant. Cette cécité inattentionnelle aurait pour conséquence, que même lorsqu'un conducteur regarde directement un objet, il pourrait ne pas le voir ( «look but fail to see ») parce que son attention est détournée vers un contexte autre que celui de la conduite.

L'enregistrement de l'activité électrique cérébrale (EEG) et plus précisément des potentiels évoqués par des événements de la scène routière, a permis à ces auteurs [15-16] de mieux comprendre les mécanismes mis en jeu. La diminution de l'amplitude d'ondes telles que P300 pendant les conversations téléphoniques témoignerait d'une interférence au moment de l'encodage initial de l'information issue de la scène routière. En d'autres termes, converser au téléphone en conduisant affecterait la façon dont les conducteurs prêtent attention à un stimulus dans l'environnement de conduite. En utilisant la même technique, Bruyas et coll. [17] ont montré que cette baisse d'amplitude était également liée à la complexité de la tâche de communication. Plus la communication est complexe et plus elle est importante, témoignant d'une diminution également plus importante des ressources allouées à la tâche de conduite.

Un autre cadre méthodologique a été proposé par McCarley et coll. [18] pour mieux comprendre ces phénomènes, avec le paradigme de cécité au changement («change blindness »). Ces derniers ont montré qu'une conversation téléphonique naturelle pouvait perturber la détection de changements dans des scènes routières complexes statiques. Les auteurs expliquent en partie ce résultat par une altération de la recherche oculomotrice en condition téléphonique et, en accord avec Strayer et coll. [14-15], par un déficit de l'encodage de l'information au moment de la fixation.

\subsection{Effet d'une conversation téléphonique sur les performances de conduite}

Dans ce domaine, beaucoup de travaux ont été réalisés et ce, plus particulièrement au cours des deux dernières décennies. Les méta-analyses de Horrey et Wickens [19] et de Caird et coll. [20] en proposent une synthèse. Les travaux effectués dans ce domaine ont également tenté d'analyser la possibilité de comportements d'adaptation des conducteurs : augmentation des distances de suivi ou réduction des vitesses, mais les résultats obtenus sont divergents. 


\subsubsection{Temps de réponse}

Les méta-analyses citées plus haut se sont basées sur un grand nombre d'études. Toutes s'accordent pour trouver un coût significatif du téléphone sur les performances de conduite, exprimées en termes de temps de réaction à un événement ou à un stimulus. Selon ces métaanalyses, les résultats montrent de façon indiscutable que les temps de réponse des conducteurs augmentent lorsqu'ils téléphonent au volant. Horrey et Wickens [19], tout comme Caird et coll. [20] montrent également que la taille des effets est comparable, que les résultats soient obtenus sur simulateur de conduite ou en condition réelle de conduite.

Par ailleurs, Harbluk et coll. [4] ont observé, en conduite réelle, que les conducteurs effectuent un nombre plus élevé de freinages brusques lorsqu'ils sont engagés dans une tâche de calcul difficile par téléphone. Hancock et coll. [11] ont également observé, sur piste, que les conducteurs distraits freinent de façon plus brutale lorsqu'ils doivent s'arrêter à un feu rouge qu'ils ne le font lorsqu'ils ne sont pas distraits. Harbluk et coll. [4] suggèrent que la réduction du contrôle visuel de l'environnement effectuée par un conducteur qui téléphone pourrait en partie expliquer cette modification du comportement de freinage. En effet, pour freiner de façon appropriée, le conducteur doit effectuer un contrôle de l'environnement lui permettant de prendre en compte toutes les informations pertinentes. Lorsqu'il est distrait, la prise en compte de ces éléments est altérée, ce qui retarde le moment de prise de décision. Dans ce cas, un freinage plus brutal permet de compenser ce délai.

\subsubsection{Dynamique du véhicule}

Selon la méta-analyse réalisée par Caird et coll. [20], converser au téléphone n'affecte de façon sensible ni le contrôle latéral ni les distances inter-véhiculaires. En effet, l'impact est minimal et surtout diffère en fonction des études qui sont peu nombreuses et parfois contradictoires. À titre d'exemple, pour Alm et Nilsson [21], les conducteurs qui téléphonent ne compensent pas l'augmentation de leurs temps de réaction en augmentant leurs distances inter-véhiculaires ; tandis que pour Strayer et coll. [14], ils augmentent la distance à laquelle ils suivent un véhicule et ce, que le trafic soit dense ou non.

Comme le soulignent Horrey et Wickens [19], le fait que l'impact du téléphone soit plutôt exprimé en termes d'augmentation des temps de réponse, qu'en termes de contrôle de la trajectoire s'explique. En effet, le contrôle de la trajectoire est une habileté relativement automatique qui nécessite peu de ressources attentionnelles. Répondre à un signal est moins automatique car le conducteur ne doit pas seulement détecter ce signal, mais aussi sélectionner une séquence d'actions appropriées pour y répondre. Notons, toutefois, que certaines études ont montré que le contrôle latéral pourrait être amélioré pendant les communications téléphoniques [9][12][22]. En effet, le contrôle de la trajectoire est fortement lié à la direction des regards. La concentration spatiale des regards vers le centre de la voie, observée lorsque les conducteurs effectuent des tâches cognitives, pourrait ainsi entraîner, dans certains cas, un meilleur maintien du véhicule sur la voie.

L'éventualité que les conducteurs compensent l'augmentation des temps de réponse engendrée par une conversation téléphonique en réduisant leurs vitesses a été également évaluée. Ici encore les résultats sont divergents. Si certaines études montrent une réduction des vitesses lorsque les conducteurs conversent avec un téléphone mains-libres [23-26], d'autres ne font apparaître une réduction des vitesses que si le téléphone est tenu à la main [12] [27-29]. Le trafic pourrait également jouer un rôle : la vitesse ne diminue que lorsque le trafic est modéré à fort et non s'il est faible [26]; ainsi que la durée de l'appel : diminuant pendant les appels courts, la vitesse augmenterait quand ils sont plus longs [30]. 


\subsubsection{Prise de décision}

Très peu de travaux se sont intéressés aux effets de l'utilisation du téléphone sur les aspects plus tactiques ou stratégiques et sur la prise de décision. Les premiers travaux qui ont montré l'effet délétère d'une conversation téléphonique sur les capacités d'un conducteur à prendre une décision ont été réalisés par Brown et col. [31]. Les conducteurs devaient passer des portes de différentes largeurs. Lorsqu'une porte était jugée trop étroite, ils empruntaient une branche de contournement. Les erreurs d'appréciation se sont avérées bien plus nombreuses pendant les phases de communications téléphoniques.

Plus tard, les interactions entre des conducteurs en train de téléphoner et les autres usagers de la route ont été observées en conduite réelle par Anttila et Luoma [32]. Ces auteurs ont montré que réaliser une tâche auditive perturbe la façon dont les conducteurs interagissent avec les autres usagers notamment en intersection (augmentation des attentes non nécessaires avant de s'engager, augmentation des comportements inappropriés envers les usagers vulnérables, les contraignant à s'arrêter pour éviter un conflit, par exemple). Cooper et coll. [26] ont exploré l'influence d'une conversation téléphonique sur des changements de voie non contraints. Le nombre de changements de voie s'est avéré significativement moins élevé pendant les conversations, notamment lorsque le trafic était modéré à fort. Des résultats similaires ont été obtenus par Beede et Kass [33]. Ces derniers ont montré, en outre, que les conducteurs commettaient un nombre significativement plus élevé de violations et d'erreurs attentionnelles (inspection insuffisante d'une intersection, arrêt en l'absence de stop ou à un feu vert ou démarrage avant un feu vert) pendant les conversations téléphoniques. En prenant moins souvent la décision de changer de voie, les conducteurs privilégient leur trajectoire ; ils évitent les sous-tâches de la conduite plus secondaires afin d'allouer plus de ressources attentionnelles à la double tâche de téléphoner et conduire. Même si ce phénomène peut s'apparenter à une adaptation positive de la conduite, Beede et Kass (2006) soulignent qu'un comportement qui consiste à éviter la réalisation de certaines tâches mais au cours duquel est enregistré un nombre plus important d'erreurs et de violations plaide en faveur d'une altération de la conscience de la situation, parce que les conducteurs ne parviennent plus à traiter toutes les informations de l'environnent routier.

L'utilisation du téléphone affecterait aussi davantage les conducteurs dans les situations exigeant une prise de décision complexe, comme celle de tourner à gauche, et moins dans des situations où les prises de décision sont plus simples, comme celle de s'arrêter à un feu rouge [34]. Selon ces auteurs, son impact serait aggravé lorsque les conditions de conduite sont mauvaises (route mouillée), empêchant les conducteurs de réaliser les ajustements nécessaires pour gérer correctement l'interaction avec les autres véhicules; les prises de décision s'avèrent alors plus risquées.

\subsection{Comparaison avec d'autres activités verbales et auditives}

L'impact d'une conversation téléphonique sur le comportement de conduite a été comparé à la pratique d'autres activités verbales ou auditives telles que l'écoute de la radio ou le fait de discuter avec un passager. Il en ressort que toutes les tâches auditives n'altèrent pas les performances de conduite de la même manière.

\subsubsection{Téléphoner et écouter la radio}

Plusieurs études ont comparé l'effet de l'écoute de la radio avec celui d'une conversation téléphonique. Contrairement à la condition téléphonique, aucune influence sur les temps de réponse n'est constatée lorsque les conducteurs écoutent la radio [10][17][35]. Pour Recarte et Nunes [6], seules les tâches qui impliquent la production d'une réponse verbale ont un effet sur la recherche visuelle et sur les capacités de détection et de sélection de la réponse. Pour 
ces auteurs, recevoir une information sous la forme de messages neutres, sans connotation émotionnelle, et ne nécessitant pas la réalisation d'une action immédiate ne perturbe pas la tâche de conduite. Ainsi, écouter un matériel vocal n'est pas suffisant, en soi, pour générer une interférence avec la tâche de conduite.

\subsection{2. Écoute vs production verbale}

Plus récemment, Kunar et coll. [36] ont testé différentes conditions de production de parole. Ils ont montré que si une conversation téléphonique altère bien les temps de réponse à une tâche dite de tracking consistant à poursuivre des cercles en mouvement, il en est de même lorsque des sujets génèrent des mots à partir de mots entendus. À l'inverse, les temps de réponse ne sont altérés ni lors de l'écoute d'un récit ni lors d'une tâche de répétition de mots. Ces auteurs en concluent que l'interférence observée ne se situe pas au niveau moteur de la production du langage, mais au niveau des processus cognitifs nécessaires à la conduite d'une discussion. Seules les tâches les plus complexes interfèrent et non les tâches purement motrices comme celles de répéter des mots.

De tels résultats peuvent être rapprochés de ceux obtenus par Bruyas et coll. [13], qui ont évalué l'effet d'une communication rendue asynchrone grâce à l'usage d'un répondeur. La communication est ainsi segmentée en trois phases : écoute du message, production de la réponse et interactions vocales avec le système. Les phases les plus perturbatrices se sont avérées les phases de production d'une réponse verbale, au cours desquelles l'engagement du conducteur est le plus important. À noter que l'usage d'un tel répondeur pourrait se révéler avantageux en conduite, parce qu'il place la communication sous le contrôle du conducteur qui peut réécouter le message et choisir un moment adéquat pour y répondre, ce qui n'est pas le cas d'une conversation téléphonique. Notons encore que les phases de réponse se sont avérées très courtes, ce qui confère à un répondeur de ce type un avantage additionnel, sur une conversation téléphonique.

\subsubsection{Discuter au téléphone ou avec un passager}

Il convient, en premier lieu, de rappeler qu'une conversation quelle qu'elle soit place le conducteur en situation de double tâche, ce qui a de fait un effet potentiel sur la conduite. À cet égard, la méta-analyse de Caird et coll. [20] fait apparaître un coût à peu près équivalent en termes de temps de réponse entre les deux tâches.

Pour Drews et coll. [37], les deux types de conversations diffèrent parce que le trafic environnant peut devenir un sujet de la conversation, ce qui aide passager et conducteur à partager une même conscience de la situation et par conséquent atténue les effets négatifs de la conversation sur la tâche de conduite. Crundall et coll. [38] ont étayé cette hypothèse en montrant que le rythme d'une conversation avec passager se modifie en fonction des exigences de la route, un phénomène qu'ils ont appelé suppression de la conversation. Conducteur et passager interrompent naturellement la conversation lorsque la situation de conduite se complexifie, ce qui ne peut se produire lors d'un appel téléphonique.

Par ailleurs, Drews et coll. [37] ont observé un effet différent sur la conduite des deux types de conversation : notamment une plus grande variabilité de la trajectoire, de plus grandes distances inter-véhiculaires, et un plus grand nombre d'erreurs de navigation pendant les conversations téléphoniques que pendant les conversations avec passager. Ils expliquent ce dernier résultat, en faisant référence à l'hypothèse de cécité inattentionnelle [14-16]. Les conducteurs traiteraient insuffisamment les informations provenant de l'environnement lorsqu'ils téléphonent, alors que lorsqu'ils discutent avec un passager, ce dernier serait susceptible de suppléer à cette insuffisance. Pour Hunton et Rose [39], tout comme pour Charlton [40], les conversations téléphoniques sont associées à un nombre plus important d'erreurs de conduite et «d'accidents » que celles avec un passager. Notons qu'il s'agissait 
dans les deux cas d'expérimentations sur simulateur de conduite. Pour ces auteurs, les conversations téléphoniques exigent davantage de ressources attentionnelles de la part du conducteur, ce qui est préjudiciable pour la tâche de conduite.

Pour tenter de mieux comprendre les différences entre les deux types de conversations, plusieurs études ont analysé les variations des conversations dans les deux conditions. Lorsque l'on conduit, une dégradation plus importante de la qualité du discours est observée pendant les conversations téléphoniques comparées à des conversations avec un passager. Moins de mots sont prononcés par minute [41], tandis que les nombres d'hésitations et de répétitions sont plus élevés [42], ainsi que le nombre d'erreurs [43]. La complexité de la tâche de conduite a également un impact négatif sur la qualité du discours : la longueur moyenne des énoncés diminue [38], le nombre des hésitations augmente [42], ainsi que le nombre de répétitions [43], la complexité du discours exprimée en termes de syllabes par mot diminue [37][43].

Le fait que le discours soit davantage altéré pendant les conversations téléphoniques pourrait être révélateur d'une demande attentionnelle plus importante de la tâche de communication téléphonique. Deux raisons peuvent l'expliquer. La première est liée à l'absence de l'interlocuteur, ce qui exige des ressources cognitives supplémentaires de la part du conducteur qui doit compenser l'absence d'indices non verbaux existants dans une situation de face à face [44]. Parallèlement, si une conversation avec un passager peut être rythmée par le conducteur et interrompue lorsque la demande attentionnelle de la conduite augmente [38], le rythme d'une conversation téléphonique est dirigé par une attente de continuité, un silence étant potentiellement mal compris par l'interlocuteur [42].

\subsection{Téléphone mains-libres ou tenu à la main}

Plusieurs études ont tenté de comparer les effets sur la conduite d'un téléphone tenu à la main et d'un téléphone mains-libres. Tout d'abord, pour Patten et coll. [28] et Törnros et Bolling [12], le fait de maintenir une conversation au téléphone en conduisant induit une augmentation de la charge mentale, mais celle-ci est comparable quel que soit le téléphone utilisé ; alors que pour Burns et coll. [27], les conducteurs jugeraient la demande attentionnelle supérieure lorsque le téléphone est tenu à la main que lorsque c'est un téléphone mains-libres.

Les performances de conduite ont été évaluées, tout d'abord, en termes de temps de réponse. Comme précédemment, ces derniers augmentent lorsque le conducteur téléphone au volant, mais aucune différence n'est enregistrée que le téléphone soit mains-libres ou tenu à la main [10][27][35][45-46]; ce que confirment les méta-analyses de Horrey et Wickens [19] et de Caird et coll. [20].

C'est en termes de vitesses que les différences entre les deux types de téléphone sont les plus importantes. Plusieurs études ont notamment montré que les conducteurs réduisaient significativement leur vitesse, lorsqu'ils utilisent un téléphone est tenu à la main [12][27-29]. Cette réduction des vitesses pourrait traduire une adaptation du comportement de conduite visant à réduire la charge mentale engendrée par le fait de téléphoner en conduisant afin de la maintenir à un niveau acceptable ; ce qui expliquerait, au final, que la charge mentale soit comparable dans les deux cas. Cependant, s'il est important de souligner la possibilité de la mise en place d'un tel comportement d'adaptation, Patten et coll. [28] insistent sur le fait qu'il n'est peut-être pas suffisant pour compenser la réduction d'attention accordée à la tâche de conduite. Une autre explication réside dans le fait que les conducteurs pourraient avoir davantage conscience des effets négatifs sur la conduite d'une distraction engendrée par une tâche manuelle, telle que tenir son téléphone à la main, et sous-estimer cette distraction si elle est uniquement cognitive avec un téléphone mains-libres. 
De ces résultats, il ressort que les effets d'une conversation par le biais d'un téléphone tenu à la main versus mains-libres sur le comportement de conduite ne semblent pas si différents, qu'ils soient exprimés en termes de charge mentale, ou en termes de comportement de conduite, à l'exception des vitesses qui sont parfois réduites dans le cas du téléphone tenu à la main. S'il est clair qu'un kit mains-libres ne peut pas résoudre tous les problèmes attentionnels liés à l'utilisation du téléphone au volant, l'impact négatif du téléphone tenu à la main pourrait toutefois être exacerbé dans les situations qui nécessitent une intervention manuelle de la part du conducteur (tourner en intersection, par exemple).

\subsection{Converser et manipuler son téléphone}

Outre le fait de discuter avec un interlocuteur, utiliser un téléphone portable sous-entend la réalisation de diverses tâches, telles que numéroter, décrocher/raccrocher. D'autres fonctions sont également disponibles, telles que lire ou écrire des SMS, ou encore consulter les nombreux services disponibles sur Internet... Les travaux mentionnés précédemment étaient focalisés sur l'effet de tâches cognitives telles que converser au téléphone et en excluaient la manipulation proprement dite. Les tâches de nature visuo-manuelle auront, bien évidemment, des répercussions bien différentes sur la conduite.

\subsubsection{Effet des tâches visuo-manuelles sur la conduite}

Une tâche visuelle induit nécessairement un détournement du regard vers le dispositif utilisé, entraînant de fait l'interruption momentanée du traitement des informations en provenance de l'environnement routier. Victor et coll. [8] ont montré que, lorsque l'on conduit, le temps passé à regarder un dispositif sur lequel des informations sont affichées s'accroît avec la complexité de la tâche visuelle à réaliser : les regards sont plus nombreux, durent en moyenne plus longtemps et le nombre de regards dépassant les deux secondes augmente.

La réalisation de tâches visuo-manuelles a également des répercussions différentes sur les performances de conduite. Tout d'abord, on observe une augmentation plus importante des temps de réponse pour détecter des signaux ainsi qu'une diminution plus importante du nombre de signaux correctement détectés pendant les phases de numérotation comparées à des phases de conversations téléphoniques [12].

Les répercussions des tâches visuo-manuelles s'avèrent particulièrement négatives, en termes de contrôle de la trajectoire. Briem et Hedman [47] ont montré une augmentation des déviations de trajectoires pendant la manipulation des commandes de la radio. Des résultats comparables sont obtenus pendant les phases de numérotation, par Törnros et Bolling [12] pendant la réalisation de différentes tâches visuo-manuelles incluant des tâches de numérotation, par Ranney et coll. [48] et pour Tsimhoni et coll. [49], en ce qui concerne des entrées de destinations sur un système de navigation. Pour Jamson et Merat [50], l'altération de la trajectoire serait d'autant plus importante que la tâche visuo-manuelle est complexe.

Comme l'ont montré Engström et ses collaborateurs [9], la direction des regards est fortement liée au contrôle de la trajectoire, et plus le conducteur quitte la route des yeux, plus sa position sur la voie se dégrade. Le partage temporel nécessaire pour réaliser une tâche visuo-manuelle en conduisant induit un contrôle intermittent de l'environnement, au cours duquel le conducteur s'efforce de maintenir une trajectoire acceptable en réduisant sa vitesse et/ou en faisant de larges corrections avec son volant; contrairement aux tâches auditives qui entraînent une concentration des regards vers le centre de la voie et sont associées à de meilleures performances de maintien sur la voie. C'est à ce niveau que se révèlent les différences les plus importantes entre les effets sur les performances de conduite d'une tâche visuo-manuelle et ceux d'une tâche cognitive ou auditive. Caird et coll. [20] soulignent également que la tenue et/ou la manipulation du téléphone ou d'un clavier nécessite l'usage 
d'une main, ce qui peut générer une interférence biomécanique avec la tenue du volant et ajouter aux difficultés du contrôle de la trajectoire.

Toutefois, différents auteurs ont mis en évidence certaines formes d'adaptation des conducteurs à ces situations d'attention partagées, telles qu'une réduction de la vitesse pour manipuler les commandes de l'autoradio ou insérer une cassette [51] ou une augmentation des distances inter-véhiculaires [48-49]. De tels comportements visant à réduire la vitesse ou à augmenter les distances inter-véhiculaires sont interprétés comme des tentatives de la part des conducteurs pour réduire la demande attentionnelle additionnelle engendrée par la réalisation de ces tâches visuo-manuelles.

\subsubsection{Envoi et réception de SMS}

Peu d'études se sont focalisées sur l'effet sur la conduite de l'envoi ou de la réception de SMS [52-53]. Pour Hosking et coll. [53], lire et écrire des SMS perturbent fortement l'exploration visuelle de l'environnement routier : les conducteurs regardent plus souvent et deux fois plus longtemps à l'intérieur du véhicule qu'en condition de référence (sans SMS). Ce temps d'absence de contrôle de l'environnement routier a, bien évidemment, d'importantes conséquences en termes de performances de conduite: notamment une altération de la trajectoire et une plus grande variabilité de la position latérale qu'en condition de référence. Les conducteurs compensent toutefois ces difficultés en augmentant leur distance de suivi. Les travaux de Drews et coll. [52] ne font que confirmer ces résultats. Toutefois pour ces derniers, si une augmentation des distances inter-véhiculaires moyennes est bien constatée, une plus grande variabilité de ces distances ainsi que des distances minimales plus courtes sont également enregistrées. Comparant ces résultats avec des données obtenues précédemment dans les mêmes conditions avec des conversations téléphoniques, Cooper et Strayer [54] montrent une altération des performances de conduite bien plus importante pour la lecture et la rédaction de SMS, que pour une conversation téléphonique. Ils en concluent que lire et écrire des SMS en conduisant pourraient être plus dangereux que bien d'autres activités distractives dans lesquelles un conducteur peut s'engager.

\section{2. Études épidémiologiques}

Téléphoner en conduisant modifie-t-il le risque d'avoir un accident, et dans quelle mesure ? Si une augmentation du risque est avérée, dépend-elle du système utilisé (mains-libres ou non) ? Dans l'ensemble des accidentés, quelle part parmi ceux-ci peut-on attribuer au «téléphone au volant »? Selon la pratique courante en épidémiologie, la réponse à la première question passe par le calcul du risque relatif (RR) estimé en divisant le risque pour un conducteur d'être accidenté en téléphonant par le risque d'être accidenté sans téléphoner.

$$
\mathrm{RR}=\frac{\text { Risque d'être accidenté en téléphonant }}{\text { Risque d'être accidenté sans téléphoner }}
$$

L'évaluation de ce rapport RR suppose la connaissance, pendant une durée d'observation donnée, des périodes pendant lesquelles chaque conducteur de l'échantillon observé circulait en situation de téléphoner ou pas. Cela suppose également la connaissance des éventuels accidents survenus au cours des mêmes périodes. Le nombre assez faible d'études épidémiologiques disponibles dans la littérature internationale et pertinentes sur le sujet s'explique par la difficulté de disposer de ces informations sur des échantillons suffisamment importants. Il faut également souligner une difficulté supplémentaire liée à l'intermittence des expositions à la conduite et au téléphone. Contrairement à beaucoup d'expositions examinées en santé publique, l'effet de l'utilisation du téléphone est transitoire (l'effet de perturbation 
sur la conduite est supposé cesser dès ou peu de temps après l'arrêt d'utilisation du téléphone). Ceci nécessite d'avoir une connaissance précise des différentes périodes d'exposition et de l'instant de l'accident. Il faut de plus que le RR soit le plus possible interprétable en tant qu'effet propre de l'utilisation du téléphone, ce qui s'obtient à la fois en choisissant un type d'étude adapté et par un ajustement sur les autres facteurs pouvant jouer simultanément sur la survenue de l'accident et sur l'usage du téléphone. À défaut de pouvoir estimer directement le RR, beaucoup d'études (tableau 5.I) estiment l'odds ratio (OR) qui en est une bonne approximation dans le cas où l'événement d'intérêt est « rare ». Ceci est le cas quand on s'intéresse à la survenue d'un accident, qui est un événement rare pour un conducteur.

Les publications retenues dans cette revue de la littérature épidémiologique concernent, d'une part, les recherches apportant des résultats significatifs [55-66] et, d'autre part, celles relatives à des considérations méthodologiques permettant un regard critique sur certaines d'entre elles [57][67-78]. Quatre revues récentes de la littérature [79-82] qui s'intéressent à l'effet du téléphone en conduisant, ou plus largement aux facteurs de "distraction", ont également été prises en compte.

\subsection{Principaux résultats et estimations du risque d'accident associé à l'usage du téléphone au volant}

Les études de Wilson et coll. [62], Sullmann et Baas [63] et Laberge-Nadeau et coll. [61] répondent à la question «les possesseurs de téléphone ont-ils plus tendance à avoir des accidents que les autres ? ». Elles ont été réalisées à une époque de faible taux d'équipement, et les personnes qui possédaient alors un téléphone portable avaient certainement des caractéristiques socio-économiques différentes des autres, et donc des façons différentes d'utiliser leurs véhicules. Les auteurs de ces travaux ont produit des estimations des risques relatifs ajustées sur une partie de ces caractéristiques. Ainsi, pour tenter d'isoler l'effet propre du téléphone, les estimations des odds-ratios sont ajustées, entre autres, sur le sexe, l'âge et le kilométrage parcouru dans les études de Laberge-Nadeau [61] et de Sullmann [63]. Comme attendu, cet ajustement fait baisser les estimations brutes de départ, et les sur-risques trouvés sont faibles, entre 1,10 et 1,20, mais significativement différents de 1 . L'étude de LabergeNadeau montre de plus que les conducteurs utilisant fréquemment le téléphone avaient un risque d'accident plus élevé que ceux qui l'utilisent peu, ce qui constitue un élément en faveur d'une possible interprétation causale. Dans l'étude de Wilson [62], l'OR estimé est du même ordre de grandeur alors qu'il n'est pas ajusté sur le kilométrage mais sur un indicateur de conduite dite agressive, défini à partir des infractions relevées. Autre différence, il compare des conducteurs considérés «en faute» à des conducteurs qui sont considérés comme impliqués par «manque de chance», constituant en cela des témoins de conducteurs circulants. Si l'inconvénient de la démarche est de passer par la définition d'une responsabilité souvent difficile à établir, elle présente l'avantage de comparer les conducteurs « en faute » à des conducteurs témoins soumis aux mêmes conditions de circulation quand ils sont impliqués dans le même accident.

De ces différentes études, on peut retenir que le risque d'accident associé à la possibilité de téléphoner au volant est compris entre 1,10 et 1,20, sachant qu'il s'agit d'une valeur moyenne pour l'ensemble des conducteurs qui utilisent leur téléphone au volant, certains ne l'utilisant que très rarement, d'autres une grande partie de leurs trajets. L'étude de Nabi et coll. [65] apporte une précision supplémentaire en distinguant, d'une part, les conducteurs qui déclarent n'utiliser le téléphone au volant que quand les conditions de conduite s'y prêtent et, d'autre part, les conducteurs déclarant l'utiliser quelles que soient les circonstances. Le risque de 1,7 associé aux deuxièmes comparé aux non utilisateurs, concerne moins de $10 \%$ des utilisateurs 
de téléphone. Si l'on considère que les premiers n'ont pas d'augmentation significative du risque d'accident par rapport aux non utilisateurs, la valeur du risque associée à l'ensemble des utilisateurs de téléphone, c'est-à-dire 1,7x10\%+1x90\%, soit 1,07, est du même ordre de grandeur que dans les études précédentes.

$\mathrm{Au}$ contraire des précédentes recherches, les études en case crossover de Redelmeier et Tibshirani [56-57] et de McEvoy et coll. [64] s'attachent à estimer le risque d'accident pour un conducteur en train de téléphoner relativement à un conducteur ne téléphonant pas. Il est donc naturel que les risques trouvés soient plus élevés que les précédents, compris entre 3 et 5 selon les études et les sous-groupes étudiés. À noter que ces études ne font pas la différence entre les conversations téléphoniques et l'envoi de SMS. Cette dernière pratique était quasi inexistante à l'époque du recueil des données de l'étude canadienne, et encore peu courante pour l'étude australienne, et cet amalgame n'a sans doute que peu d'effet sur les résultats.

Le principal problème lié à ce type d'étude est le risque d'attribution des appels téléphoniques survenus après l'accident à la période précédant l'accident, en raison de la connaissance imprécise de l'instant de l'accident. Cette confusion entre la cause et l'effet prêterait d'autant plus à conséquence que toutes les études montrent que le nombre d'appels après l'accident est très élevé. Dans l'étude de McEvoy, par exemple, la moitié des accidentés utilisent leur téléphone après l'accident. De plus, et bien qu'il s'agisse d'accidents corporels, les appels s'adressent principalement à des membres de la famille (65\%), à des amis, au lieu de travail, les appels aux services d'urgence $(31 \%)$ venant ensuite. Ceci signifie que la précaution d'exclure les appels d'urgence est nécessaire mais n'est pas suffisante pour éviter complètement de prendre en compte les appels dus à l'accident. Cependant, les études de Redelmeier et de McEvoy ont tout fait pour éviter ce biais de classement : les deux études excluent les appels d'urgence et ne conservent les conducteurs qu'en cas de cohérence entre les sources d'information concernant l'instant de l'accident. Surtout, l'OR attaché aux appels entrants est le même que celui attaché aux appels sortants pour Redelmeier, ce qui est un argument très fort pour penser que ce biais de classement a été évité en grande partie.

Un autre point à considérer est que l'association statistique mise en évidence peut ne pas être causale : les conditions de conduite (le mauvais temps, une conduite sous forte contrainte temporelle ou émotionnelle...) peuvent contribuer à la fois à la survenue d'un accident et à l'usage du téléphone. Le fait de comparer un conducteur à lui-même permet d'ajuster les estimations de risques sur les caractéristiques «stables » d'un individu, mais ne permet pas d'ajuster sur les conditions de conduite qui peuvent être différentes entre les périodes comparées, aussi bien concernant l'environnement du conducteur que ses facultés du moment. Il est par exemple impossible d'ajuster sur l'alcoolémie, qui ne peut être estimée qu'au moment de l'accident, alors que le fait de conduire sous l'emprise alcoolique peut avoir une influence à la fois sur le risque d'accident et sur l'utilisation du téléphone. Là encore, le résultat de Redelmeier ne prenant en compte que les appels entrants est d'une grande importance. Et bien sûr, la vraisemblance d'une relation causale vient des résultats des études expérimentales qui donnent une explication au mécanisme créant une perturbation de l'attention défavorable à la tâche de conduite.

Enfin, la méthode appliquée consiste à comparer des périodes pendant lesquelles le conducteur a déclenché ou reçu un appel, sans savoir s'il téléphonait encore lors de l'accident. L'éventualité d'une mauvaise classification introduit un biais non différentiel si l'on admet que l'erreur de classement est la même pour les périodes juste avant l'accident et les périodes témoins. Ce biais entraîne vraisemblablement une sur-estimation des risques qui n'est pas discutée par les auteurs.

Avec une méthodologie très différente basée sur la comparaison des conducteurs estimés « en faute » et ceux qui ne le sont pas, Sagberg [59] arrive à une estimation du risque relatif de 2,4, qui est sans doute une sous-estimation étant donné la méthode employée. Ainsi, si l'on 
considère que les études en case crossover ont tendance à sur-estimer le risque, le choix d'une valeur autour de 3 paraît raisonnable pour la suite des calculs.

Par ailleurs, dans la plupart des travaux qui ont étudié les caractéristiques des accidents associés à l'usage du téléphone, il a été montré une légère sur-représentation des accidents avec choc arrière, le conducteur téléphonant venant percuter l'arrière d'un autre véhicule par « distraction ». Ce type d'accident pourrait présenter certaines spécificités en termes de gravité, les risques associés étant alors différents selon le critère de gravité auquel on s'intéresse (matériel, corporel, mortel). En faveur de cette hypothèse, la seule étude disponible est celle de Violenti de 1998 [58], mais elle comporte beaucoup de faiblesses. Les études de Redelmeier et de McEvoy, l'une expliquant la survenue d'accidents matériels, et l'autre d'accidents corporels, donnant des résultats proches, les risques d'être impliqués dans un accident corporel ou matériel selon qu'on téléphone au volant ou pas ne seront pas distingués. Enfin, et avant de proposer des estimations de risques à retenir, il faut noter que nous n'avons pas pris en compte les résultats de deux études : celle de Violenti [55] conduite en 1996 qui, malgré un protocole pertinent, a une puissance statistique trop faible, et l'étude de Young et Schreiner [66], qui bénéficie de données d'exposition d'une grande précision, mais qui concerne un système mains-libres produit par un fabriquant unique, qui ignore l'éventuelle utilisation d'un téléphone portable personnel et qui présente des estimations de risques non ajustées sur des facteurs importants.

Par ailleurs, si cette dernière étude pourrait être avancée comme un argument en faveur des systèmes mains-libres, aucune des études épidémiologiques qui comparent les deux modes d'utilisation ne montre une différence significative entre le risque associé au téléphone tenu à la main et celui associé aux différents dispositifs mains-libres existants. Le téléphone tenu à la main représente un risque toujours supérieur, mais pas assez pour présenter une différence statistiquement significative dans les différentes études.

À noter également que toutes les recherches incluses portent sur l'utilisation du téléphone au volant essentiellement par des conducteurs de véhicules particuliers, plus rarement des conducteurs de poids lourds ou de véhicules utilitaires. Aucune ne porte sur l'effet du téléphone sur la conduite pour des conducteurs d'autres types de véhicules ou sur le comportement des piétons en traversée de chaussée. La plupart de ces études ont été réalisées avant l'entrée en vigueur de certaines lois pénalisant l'usage du téléphone tenu à la main.

\subsection{Estimation du risque relatif et du risque attribuable à l'usage du téléphone au volant}

Finalement, deux estimations de risques relatifs peuvent être retenues :

- la première, entre 1,1 et 1,2, représente le risque moyen d'accident pour un conducteur susceptible de téléphoner au volant, autrement dit téléphonant pendant une partie de son temps de conduite ;

- la deuxième estimation, autour de 3 , représente le risque d'être impliqué dans un accident matériel ou corporel pour un conducteur téléphonant par rapport à un conducteur ne téléphonant pas, ceci quel que soit le système utilisé (mains-libres ou non). C'est le sur-risque pris par le conducteur au moment où il téléphone dans son véhicule.

Dès lors, il est important de savoir quelle proportion de ses trajets est concernée par cette augmentation de risque.

Deux façons d'approcher cette valeur sont employées. D'après un certain nombre d'enquêtes réalisées dans différents pays, entre 2 et $6 \%$ des conducteurs ont été aperçus en train de téléphoner au volant. Ce chiffre est évidemment dépendant de plusieurs facteurs, tels que le 
taux d'équipement en téléphone qui a considérablement évolué, la loi en vigueur plus ou moins respectée ou le taux d'équipement en systèmes mains-libres qui échappent à l'observation. D'après l'enquête réalisée en France en 2007 [83], 2,4 \% des conducteurs ont été aperçus avec un téléphone en main au volant. D'après d'autres éléments de cette enquête, $41 \%$ des conducteurs utilisant parfois le téléphone au volant le tiennent à la main, les $59 \%$ autres utilisant un système mains-libres, y compris des oreillettes. Si on fait l'hypothèse que les temps de communication sont similaires quel que soit le système utilisé, aux $2,4 \%$ de conducteurs aperçus avec le téléphone à la main correspondraient 3,5\% de conducteurs téléphonant en mains-libres. La prévalence globale de l'utilisation du téléphone au volant serait donc de l'ordre de $6 \%$ en France, en 2007.

$\mathrm{Au}$ vu de ces estimations, en moyenne pour un trajet donné, un conducteur téléphone environ $6 \%$ du temps de parcours, temps pendant lequel il multiplie son risque par 3, alors que pendant les $94 \%$ du temps restants, il est au risque de base (par définition égal à 1). Autrement dit, son risque relatif moyen est de $6 \% \times 3+94 \% \times 1$, soit 1,1 , qui est la valeur trouvée pour le risque relatif associé aux possesseurs du téléphone. Si on prend plutôt les valeurs hautes, avec un conducteur qui téléphone $10 \%$ du trajet et un risque relatif de 4 , on obtient 1,3 pour le RR moyen. Ces estimations montrent qu'il y a cohérence entre les risques relatifs estimés dans les deux grands types d'études passées en revue.

À partir de ces valeurs, on peut estimer également un risque attribuable (RA), autrement dit la proportion d'accidents qui seraient évités si l'exposition disparaissait, $\mathrm{P}_{\mathrm{E}}$ étant la prévalence de l'usage du téléphone :

$$
\mathrm{RA}=\frac{\mathrm{P}_{\mathrm{E}}(\mathrm{RR}-1)}{1+\mathrm{P}_{\mathrm{E}}(\mathrm{RR}-1)}
$$

Avec $\mathrm{P}_{\mathrm{E}}=6 \%$ et $\mathrm{RR}=3$, on obtient $\mathrm{RA}=10,5 \%$. Si l'on considère que l'accident est survenu du fait de l'utilisation du téléphone, cela signifie qu'avec ces données de prévalence, l'utilisation du téléphone au volant serait associée à environ $10 \%$ des accidents.

On peut également faire le calcul en utilisant comme valeur du RR le risque associé au possesseur de téléphone, qui peut donc potentiellement l'utiliser en conduisant, et la prévalence correspondante, $44 \%$ [84]. En prenant $R R=1,2$, on obtient $R A=8,1 \%$. Au vu des hypothèses faites sur la pratique non observée du téléphone mains-libres pour la première estimation de risque attribuable, le fait que cette deuxième estimation soit du même ordre de grandeur est un élément très important.

En conclusion, l'estimation à 3 du risque d'accident associé à l'utilisation du téléphone au volant au moment de l'accident paraît assez consistante compte tenu des résultats des études épidémiologiques et correspond également à plusieurs résultats expérimentaux. On peut également penser que ce risque est relativement indépendant des populations d'étude.

Il est bien entendu que ce risque associé au téléphone est obtenu sans distinction des différentes activités effectuées avec, comme parler au téléphone, écouter, composer un appel, atteindre le téléphone. Il a été obtenu à une période où la pratique des texto et les smartphones étaient beaucoup moins répandus, ce qui veut dire que les activités comme celle de regarder l'écran de son téléphone et composer un SMS doivent avoir une part très réduite dans cette estimation de risque. La prévalence de l'exposition au téléphone au volant est plus fluctuante dans le temps et dépendante des populations observées. Comme le montre le calcul ci-dessus, cette valeur de prévalence est déterminante dans l'estimation du nombre d'accidents ou de victimes que l'on peut associer à la pratique du téléphone au volant. 


\section{3. Études naturalistes}

Entre les études expérimentales et les études épidémiologiques, les études de suivi de flottes de véhicules et de leurs conducteurs en situation de conduite «naturelle », dites "naturalistic driving", peuvent apporter des éléments d'interprétation des risques associés au téléphone en conduisant. Quatre études américaines ont retenu notre attention.

L'étude de Klauer et coll. [85] est celle dont les résultats sont le plus souvent repris dans les revues de la littérature citées par ailleurs. Il s'agit d'une étude de suivi pendant 12 mois de 109 voitures équipées de vidéo-caméras et d'autres dispositifs enregistreurs de façon à pouvoir observer le comportement des conducteurs et la survenue d'accidents, de "presque accidents » et d'incidents. L'intérêt de ce type d'étude est qu'il permet d'observer les nombreuses tâches secondaires (la tâche principale étant la tâche de conduite) accomplies par les conducteurs, en situation réelle de conduite. Ainsi sont produits les risques d'être impliqué dans un accident ou un "presqu'accident» associés aux différentes tâches secondaires entreprises par les conducteurs. Une hiérarchie des différentes tâches est proposée, les plus risquées étant par exemple celles d'atteindre un objet éloigné, de gérer la présence d'un insecte, de regarder un objet extérieur, de lire ou de composer un numéro de téléphone sur son dispositif tenu à la main. L'odds ratio associé à la conversation téléphonique estimé dans cette étude est supérieur à 1 , mais n'est pas significatif.

L'étude de Olson et coll. [86] utilise la même méthodologie pour 203 conducteurs de poidslourds en rassemblant les données de deux études de suivi. Là aussi, un classement des tâches selon leur dangerosité est proposé, la tâche la plus risquée étant celle d'écrire un message sur son téléphone portable (risque de survenue d'un événement détériorant la sécurité multiplié par 23 par rapport au risque de base), la conversation téléphonique n'étant pas significativement estimée à risque.

Deux difficultés essentielles se posent cependant quant à l'interprétation de ces résultats. La première est que l'événement considéré n'est pas la survenue d'un accident, car le phénomène est trop rare dans les deux études pour pouvoir obtenir des estimations de risques fiables, mais l'accident ou le «presqu'accident» dans la première étude, et l'accident, le «presqu'accident », ou une déviation non intentionnelle importante de la trajectoire dans la deuxième. Si l'intention des auteurs d'augmenter la puissance statistique apparaît légitime, considérer le presque accident, voire la modification non intentionnelle de trajectoire comme des événements précurseurs d'un accident n'est pas direct, et surtout doit dépendre de nombreux facteurs dont le type d'activité secondaire. À noter qu'une étude décrivant l'effet de l'utilisation des presqu'accidents à la place des accidents vient d'être publiée par la même équipe de recherche [87], qui tend à montrer une relation positive entre les fréquences des facteurs contribuant aux accidents et aux presque accidents. Les auteurs concluent que l'utilisation des presqu'accidents à la place des accidents conduit à une sous-estimation des risques correspondants, ce qui rend la démarche opérationnelle. La deuxième difficulté est calculatoire : les auteurs ne prennent pas en compte dans les calculs de leurs intervalles de confiance la corrélation existant entre les mesures effectuées sur le même individu, ce qui produit une sous-estimation des variances et peut amener à conclure à tort que certains effets sont significatifs.

L'étude de Sayer et coll. [88] porte sur 36 véhicules et l'analyse de 1440 séquences vidéo de 5 secondes des conducteurs en situation de conduite. Cette étude évite le défaut calculatoire précisé ci-dessus, mais manque également de puissance statistique en ne portant que sur un faible nombre de conducteurs. Il est cependant intéressant de noter que l'action de téléphoner ne semble pas modifier sensiblement la tenue de la trajectoire, mais qu'elle apparaît augmenter les temps de réaction au freinage, ce qui est conforme aux résultats expérimentaux. L'étude de Hickman [89] publiée très récemment inclut un nombre de véhicules beaucoup plus élevé. L'analyse porte en effet sur 13306 véhicules suivis pendant 3 mois. 1085 
véhicules impliqués dans des accidents matériels et corporels sont observés dans l'étude, mais l'essentiel des estimations produites concerne les risques associés aux évènements définis comme critiques et non à la survenue d'accidents. Les résultats obtenus sont cohérents avec l'étude d'Olson, la composition d'un numéro ou la prise en main du téléphone ou des oreillettes étant associées à un surrisque de survenue d'un "événement de sécurité critique" (odds ratios significatifs proches de 3,5), alors que la conversation téléphonique en mains libres est associée à un risque d'événement critique significatif pour les conducteurs de cars $(\mathrm{OR}=1,3)$ et à un effet protecteur pour les conducteurs de semi-remorques $(\mathrm{OR}=0,6)$.

Les deux difficultés d'interprétation relevées pour les autres études ci-dessus existent là aussi. On peut notamment observer que parmi les 37708 événements identifiés comme pouvant provoquer un accident, seuls $1064(2,8 \%)$ sont réellement associés à la survenue d'un accident. Autrement dit, l'énorme majorité des événements considérés pour le calcul des odds ratios ne se traduisent pas en la survenue d'un accident. De plus, comme l'indiquent les auteurs eux-mêmes, la définition des niveaux de référence des risques relatifs est assez peu satisfaisante et peut conduire à leur sous-estimation. Enfin, cette étude porte sur une population très particulière, des conducteurs professionnels de cars et poids-lourds, qui ont accepté d'être sous surveillance vidéo lors de l'ensemble de leurs déplacements. La généralisation des résultats obtenus sur des conducteurs professionnels américains de véhicules lourds ne peut donc pas être directe.

En l'état actuel des publications, il apparaît que ces études naturalistes ont le grand avantage sur les études épidémiologiques classiques précédemment mentionnées de permettre une distinction des différentes tâches pouvant distraire le conducteur de sa tâche de conduite, en particulier lors de l'utilisation du téléphone (composer le numéro, converser, atteindre le téléphone, etc.), de proposer des estimations de risques de survenue d'événements critiques (dont certains peuvent être considérés associés à la survenue d'un accident) et surtout de les hiérarchiser, la composition d'un SMS apparaissant, par exemple, comme associée à un très fort risque.

Il est probable que les prochaines études faites à plus grande échelle pourront produire des estimations consistantes des différents risques relatifs d'intérêt. Ces études peuvent d'ailleurs être considérées comme des études de cohortes, qui pourraient être analysées avec la méthodologie correspondante. Si, par exemple, l'échantillonnage des périodes "contrôles" est issu d'un tirage au sort, le design est celui d'une étude "cas-cohorte", et la modélisation des rapports de risques peut être effectuée par des modèles linéaires généralisés mixtes. Ce type de modélisation permettra une analyse multivariée, tenant compte des inter-relations entre les différents facteurs de distraction, ainsi que de la corrélation des observations faites sur les mêmes conducteurs. Des réflexions méthodologiques ciblées sur ces problématiques commencent à être publiées [90-91].

Au travers des résultats publiés à ce jour, Il est encore difficile de déduire de ces études des estimations fiables de risques d'accidents associés à l'utilisation du téléphone au volant en situation réelle de conduite pour l'ensemble des usagers.

\section{Avantages et limites des approches}

Les études expérimentales sont un excellent paradigme pour étudier le comportement des conducteurs dans un environnement contrôlé. Elles ont permis de quantifier précisément l'altération de la tâche de conduite pendant une activité téléphonique. Il convient toutefois d'en signaler quelques limites. Dans la plupart de ces études, qu'elles soient réalisées sur simulateur ou en conduite réelle, la gestion de la tâche de conduite, tout comme celle des tâches distractives est souvent assurée par l'expérimentateur et non par le conducteur. Ce dernier doit suivre des instructions et n'a que peu de marges de manœuvre pour adapter son 
comportement à la situation. Notons néanmoins que des ajustements ont pu être enregistrés dans certains cas, tels qu'une diminution des vitesses ou une augmentation des inter-distances. La tâche de conduite, en elle-même, n'est pas toujours très représentative d'une conduite naturelle, notamment lorsqu'il s'agit d'expérimentations en laboratoire ou sur simulateur de conduite. En effet, sous le terme de simulateur se retrouvent des réalités bien différentes. S'agissant parfois d'une simple tâche de «tracking » avec l'approximation d'un suivi de trajectoire (préférentiellement désignées comme expérimentations en laboratoire), le terme de simulateur peut faire référence à une variété de dispositifs dont le réalisme sera, bien évidemment, plus ou moins différent. Les expérimentations sur route, bien que plus réalistes, induisent également certains biais, liés à la conduite d'un véhicule non familier ou au manque de facteurs motivationnels. Le fait que le conducteur se sachant observé tende à conduire du mieux qu'il peut et que les situations évaluées soient généralement peu critiques pourraient laisser supposer que les résultats obtenus soient parfois optimistes. Enfin, certaines tâches de communication utilisées sont très artificielles, il s'agit fréquemment de tests mathématiques ou de tests verbaux effectués par téléphone (les conversations naturelles seraient plus souvent employées dans la littérature depuis 2003 que par le passé). Notons toutefois que, selon Caird et coll. [20], les tâches cognitives artificielles et les conversations naturelles ont des effets comparables sur les temps de réaction.

Les études épidémiologiques présentent l'avantage d'être basées sur l'observation des usagers du réseau routier en conditions réelles de circulation et en particulier ceux qui ont subi un accident. Elles ne permettent pas, en revanche, d'avoir des informations précises sur le comportement du conducteur, sinon à partir d'éléments indirects recueillis souvent rétrospectivement par rapport à l'accident. Concernant le rôle du téléphone au volant, la principale difficulté est de disposer d'une information fiable sur l'utilisation effective du téléphone au moment de l'accident, et de distinguer l'effet propre du téléphone des autres facteurs liés à la fois aux comportements des conducteurs et au fait qu'ils aient un téléphone portable. Les stratégies de construction et d'analyse des différentes recherches retenues dans cette revue de la littérature sont variées et peuvent être vues comme complémentaires, sans écarter complètement les risques de biais. Et aucune d'entre elles ne permet de distinguer les phases de discussion des phases de numérotation, les risques relatifs estimés portant donc sur l'ensemble des phases d'utilisation du téléphone.

Les suivis de flottes de véhicules permettent de disposer de beaucoup plus d'informations sur les conducteurs, leurs véhicules et les situations de conduite que les études épidémiologiques. Elles présentent, en outre, l'avantage de pouvoir différencier le type d'activité téléphonique réalisé au moment de l'événement considéré et ont permis de montrer que le risque associé à la manipulation d'un téléphone était bien supérieur à celui associé à une discussion téléphonique. Toutefois, ces études n'échappent pas à certains inconvénients des études expérimentales, notamment en sélectionnant des conducteurs volontaires et se sachant "sous observation". La principale difficulté semble être néanmoins la rareté des accidents observés, ce qui oblige les investigateurs à se reporter sur des événements tels que des incidents ou des presque accidents. Cette approche implique que ces événements soient considérés comme précurseurs d'accidents, ce qui reste à valider et à quantifier pour chacun d'entre eux sur des cohortes suffisamment importantes pour pouvoir disposer à la fois de nombres d'accidents et a fortiori d'incidents en quantité suffisante. 


\section{Conclusion}

Les recherches expérimentales ont montré que téléphoner en conduisant altère le traitement de l'information routière, augmentant ainsi la probabilité de ne pas percevoir ou de percevoir tardivement un élément important de l'environnement. À cela, s'ajoutent des temps de réponse plus longs lorsqu'un événement survient et un jugement parfois altéré. Les recherches visant à montrer des comportements d'adaptation sont limitées et les quelques résultats obtenus parfois divergents. Sans doute ces aspects mériteraient-ils d'être approfondis par des recherches spécifiques. Le développement des études dites naturalistes devrait notamment permettre de pouvoir les appréhender plus aisément. Manipuler son téléphone pour numéroter, lire ou écrire des SMS, ou encore consulter des services sur Internet a un effet encore plus négatif sur la conduite. L'augmentation des temps de réaction est plus importante et ces tâches induisent un détournement du regard vers l'intérieur du véhicule qui a un impact très négatif sur le contrôle de la trajectoire. L'interférence biomécanique avec la tenue du volant, liée à la monopolisation d'une main, ajoute encore aux difficultés du contrôle de la trajectoire et concourt à montrer que réaliser ces tâches de nature visuo-manuelle en conduisant pourrait s'avérer très dangereux.

Le risque d'être impliqué dans un accident matériel ou corporel est multiplié par 3 pour un conducteur utilisant son téléphone par rapport à un conducteur qui ne l'utilise pas au moment de l'accident. Cette estimation est obtenue à partir des diverses études épidémiologiques publiées, et correspond au sur-risque moyen sans distinction du type d'utilisation du téléphone comme les phases de numérotation ou de discussion. Par ailleurs, aucune étude épidémiologique ne montre une différence significative entre le risque associé au téléphone tenu à la main et celui associé aux dispositifs mains libres, sans distinction du type de dispositif. On peut remarquer que le téléphone tenu à la main représente un risque toujours supérieur, en cohérence avec ce qu'on peut attendre au vu des résultats expérimentaux, mais pas assez pour présenter une différence statistiquement significative dans les différentes études. Finalement, c'est bien le risque moyen de 3 qui, combiné à la prévalence de l'usage dans la population des conducteurs estimée à $6 \%$ en France en 2007, permet d'évaluer à environ $10 \%$ le nombre d'accidents corporels ou matériels associés à l'usage du téléphone au volant.

En conclusion, la combinaison de ces différentes études a permis d'une part, de montrer que la tâche de conduite était altérée lorsque l'on utilise son téléphone au volant et de quantifier cette perturbation (études expérimentales) et, d'autre part, de vérifier que cette perturbation était bien susceptible d'être à l'origine d'accidents et de quantifier le risque encouru (études épidémiologiques). Les études de suivi de flottes de véhicules, tout en apportant certaines précisions, tendent à confirmer ces différents résultats.

Les trois approches sont évidemment perfectibles, en rendant plus réalistes les études expérimentales, en validant l'approche visant à se passer de l'observation de la survenue d'accidents pour les études de suivi de flottes et en rendant plus spécifiques les études épidémiologiques.

Ces trois approches s'accordent cependant pour montrer que dans l'état actuel de la connaissance, restreindre l'utilisation du téléphone en conduisant ou modifier son usage apparaît comme un véritable enjeu de santé publique. 
Remerciements : Ce papier est issu d'une revue de littérature réalisée pour la DSCR (Délégation à la Sécurité et à la Circulation Routières) publiée en 2011 dans le cadre d'une Expertise Collective INSERM : Expertise Collective INSERM-IFSTTAR «Téléphone et Sécurité routière ». Pour plus de détails sur les études mentionnées, voir les chapitres 3 (pp 35-57) et 5 (pp 81-106).

\section{Bibliographie}

1. Pachiaudi G (2001) Les risques de l'utilisation du téléphone mobile en conduisant. Synthèse $\mathrm{N}^{\circ} 39$, INRETS Collections, 62p.

2. Recarte MA, Nunes LM (2000) Effects of verbal and spatial-imagery tasks on eye fixations while driving. Journal of Experimental Psychology: Applied 6(1):31-43.

3. Recarte MA, Nunes LM (2002) Mental load and loss of control over speed in real driving. Towards a theory of attentional speed control. Transportation Research Part F: Traffic Psychology and Behaviour 5(2):111-122.

4. Harbluk JL, Noy YI, Trbovich PL, Eizenman M (2007) An on-road assessment of cognitive distraction: impacts on drivers' visual behaviour and braking performance. Accident Analysis and Prevention 39(2):372-379.

5. Pereira M, Bruyas MP, Simões A (2010) Are elderly drivers more at risk when interacting with more than one in-vehicle system simultaneously? Le Travail Humain 73(1):53-73.

6. Recarte MA, Nunes LM (2003) Mental workload while driving: effects on visual search, discrimination, and decision making. Journal of Experimental Psychology: Applied 9(2):119-137.

7. Nunes LM, Recarte MA (2002) Cognitive demands of hands-freephone conversation while driving. Transportation Research Part F: Traffic Psychology and Behaviour 5(2):133-144.

8. Victor TW, Harbluk JL, Engström JA (2005) Sensitivity of eye-movement measures to invehicle task difficulty. Transportation Research Part F: Traffic Psychology and Behaviour 8(2):167-190

9. Engström JA, Johansson EJ, Östlund J (2005) Effects of visual and cognitive load in real and simulated motorway driving. Transportation Research Part F: Traffic Psychology and Behaviour 8(2):97-120

10. Strayer DL, Johnston WA (2001) Driven to distraction: dual-Task studies of simulated driving and conversing on a cellular telephone. Psychological Science 12(6):462-466

11. Hancock PA, Lesch M, Simmons L (2003) The distraction effects of phone use during a crucial driving maneuver. Accident Analysis and Prevention 35(4):501-514

12. Törnros JE, Bolling AK (2005) Mobile phone use-effects of handheld and handsfree phones on driving performance. Accident Analysis and Prevention 37(5):902-909

13. Bruyas MP, Brusque C, Debailleux S, Duraz M, Aillerie I (2009) Does making a conversation asynchronous reduce the negative impact of phone call on driving? Transportation Research Part F: Traffic Psychology and Behaviour 12(1):12-20 
14. Strayer DL, Drews FA, Johnston WA (2003) Cell phone-induced failures of visual attention during simulated driving. Journal of Experimental Psychology: Applied 9(1):2332

15. Strayer DL, Drews FA (2007a) Multitasking in the automobile. In: Kramer AF, Wiegmann DA and Kirlik A (eds) Attention: From theory to practice. Oxford University Press, New York, pp 121-133

16. Strayer DL, Drews FA (2007b) Cell-phone-induced driver distraction. Current Directions in Psychological Science 16(3):128-131

17. Bruyas MP, Chapon A, Lelekov-Boissard T, Letisserand D, Duraz M, Aillerie I (2006) Evaluation de l'impact de communications vocales sur la conduite automobile. Recherche Transports et Sécurité 91, 99-119

18. McCarley JS, Vais MJ, Pringle H, Kramer AF, Irwin DE, Strayer DL (2004) Conversation disrupts change detection in complex traffic scenes. Human Factors 46(3):424-436

19. Horrey WJ, Wickens CD (2006) Examining the impact of cell phone conversations on driving using meta-analytic techniques. Human factors 48(1):196-205

20. Caird JK, Willness CR, Steel P, Scialfa C (2008) A meta-analysis of the effects of cell phones on driver performance. Accident Analysis and Prevention 40(4):1282-1293

21. Alm H, Nilsson L (1995) The effects of a mobile telephone task on driver behaviour in a car following situation. Accident Analysis and Prevention 27(5):707-715

22. Pereira M (2009) In-vehicle information system-related multiple task performance and driver behaviour: comparison between different age groups. Ergonomics Thesis, Technological University of Lisbon, Faculty of Human Motricity, 319p

23. Fairclough SH, Ashby MC, Roos T, Parkes AM (1991) Effects of handsfree telephone use on driving behaviour. Proceedings of the Isata Symposium, Florence, Italy

24. Haigney DE, Taylor RG, Westerman SJ (2000) Concurrent mobile (cellular) phone use and driving performance: task demand characteristics and compensatory processes. Transportation Research Part F: Traffic Psychology and Behaviour 3(3):113-121

25. Rakauskas ME, Gugerty LJ, Ward NJ (2004) Effects of naturalistic cell phone conversations on driving performance. Journal of Safety Research 35(4):453-464

26. Cooper JM, Vladisavljevic I, Medeiros-Ward N, Martin PT, Strayer DL (2009) An investigation of driver distraction near the tipping point of traffic flow stability. Human Factors 51(2):261-268

27. Burns PC, Parkes A, Burton S, Smith RK, Burch D (2002) How dangerous is driving with a mobile phone? Benchmarking the impairment to alcohol. TRL Report, 547, Crowthorne, United Kingdom

28. Patten CJD, Kircher A, Oestlund J, Nilsson L (2004) Using mobile telephones: cognitive workload and attention resources allocation. Accident Analysis and Prevention 36(3):341350

29. Törnros JE, Bolling AK (2006) Mobile phone use--Effects of conversation on mental workload and driving speed in rural and urban environments. Transportation Research Part F: Traffic Psychology and Behaviour 9(4):298-306

30. Rosenbloom T (2006) Driving performance while using cell phones: an observational study. J Safety Res 37(2):207-212 
31. Brown ID, Tickner AH, Simmonds DCV (1969) Interference between concurrent tasks of driving and telephoning. Journal of Applied Psychology 53(5):419-424

32. Anttila V, Luoma J (2005) Surrogate in-vehicle information systems and driver behaviour in an urban environment: A field study on the effects of visual and cognitive load. Transportation Research Part F: Traffic Psychology and Behaviour 8(2):121-133

33. Beede KE, Kass SJ (2006) Engrossed in conversation: the impact of cell phones on simulated driving performance. Accident Analysis and Prevention 38(2):415-421

34. Cooper PJ, Zheng Y, Richard C, Vavrik J, Heinrichs B, et al (2003) The impact of handsfree message reception/response on driving task performance. Accident Analysis and Prevention 35(1):23-35

35. Consiglio W, Driscoll P, Witte M, Berg WP (2003) Effect of cellular telephone conversations and other potential interference on reaction time in a braking response. Accident Analysis and Prevention 35(4):495-500

36. Kunar MA, Carter R, Cohen M, Horowitz TS (2008) Telephone conversation impairs sustained visual attention via a central bottleneck. Psychonomic Buletinl Review 15(6):1135-1140

37. Drews FA, Pasupathi M, Strayer DL (2008) Passenger cell phone conversations in simulated driving. Journal of Experimental Psychology Applied 14(4):392-400

38. Crundall D, Bains M, Chapman P, Underwood G (2005) Regulating conversation during driving: a problem for mobile telephones? Transportation Research Part F: Traffic Psychology and Behaviour 8(3):197-211

39. Hunton J, Rose JM (2005) Cellular telephones and driving performance: The effects of attentional demands on motor vehicle crash risk. Risk Analysis 25(4):855-866

40. Charlton SG (2009 Driving while conversing: cell phones that distract and passengers who react. Accident Analysis and Prevention 41(1):160-173

41. Gugerty L, Rakauskas M, Brooks J (2004) Effects of remote and in-person verbal interactions on verbalisation rates and attention to dynamic spatial scenes. Accident Analysis and Prevention 36(6):1029-1043

42. Bruyas MP, Taffin M (2009) Is there any difference between conversing by phone and conversing with a passenger while driving? Proceedings of the First International Conference on Driver Distraction and Inattention, A57-P, Gothenburg, Sweden, 28-29 Septembre

43. Laberge J, Scialfa C, White C, Caird J (2004) Effects of passenger and cellular phone conversations on driver distraction. Transportation Research Record: Journal of the Transportation Research Board, TRB 1899:109-116

44. Alibali MW, Heath DC, Myers HJ (2001) Effects of visibility between speaker and listener on gesture production: some gestures are meant to be seen. Journal of Memory and Language 44(2):169-188

45. Strayer DL, Drews FA, Crouch DJ (2006) A comparison of the cell phone driver and the drunk driver. Human Factors 48(2):381-391

46. Hendrick JL, Switzer JR (2007) Hands-free versus hand-held cell phone conversation on a braking response by young drivers. Perceptual and Motor Skills 105(2):514-522 
47. Briem V, Hedman LR (1995) Behavioural effects on mobile telephone use during simulated driving. Ergonomics 38(12):1536-2562

48. Ranney TA, Harbluk JL, Noy YI (2005) Effects of voice technology on test track driving performance: implications for driver distraction. Human Factors 47(2):439-454

49. Tsimhoni O, Smith D, Green P (2004) Address entry while driving: speech recognition versus a touch-screen keyboard. Human Factors 46(4):600-610

50. Jamson AH, Merat N (2005) Surrogate in-vehicle information systems and driver behaviour: Effects of visual and cognitive load in simulated rural driving. Transportation Research Part F: Traffic Psychology and Behaviour 8(2):79-96

51. Horberry T, Anderson J, Regan MA, Triggs TJ, Brown J (2006) Driver distraction: the effects of concurrent in-vehicle tasks, road environment complexity and age on driving performance. Accident Analysis and Prevention 38(1):185-191

52. Drews FA, Yazdani H, Godfrey CN, Cooper JM, Strayer DL (2009) Text messaging during simulated driving. Human Factors 51(5):762-770

53. Hosking SG, Young KL, Regan MA (2009) The effects of text messaging on young drivers. Human Factors 51(4):582-592

54. Cooper JM, Strayer DL (2008) Effects of simulator practice and real-world experience on cell-phone-related driver distraction. Human Factors 50(6):893-902

55. Violenti JM, Marshall JR (1996) Cellular phones and traffic accidents: An epidemiological approach. Accident Analysis and Prevention 28:265-270

56. Redelmeier D, Tibshirani R (1997a) Association between cellular-telephone calls and motor vehicle collisions. N Engl J Med 336:453-458

57. Redelmeier D, Tibshirani R (1997b) Interpretation and bias in case-crossover studies. J Clin Epidemiol 50:1281-1287

58. Violenti JM (1998) Cellular phone and fatal traffic collisions. Accid Anal Prev 30:519524

59. Sagberg F (2001) Accident risk of car drivers during mobile telephone use. Int J Vehicle Design 26:57-69

60. Backer-Grondahl A, Sagberg F (2011) Driving and telephoning; relative accident risk when using hand-held and hands-free mobile phones. Safety Science 49:324-330

61. Laberge-Nadeau C, Maag U, Bellavance F, Lapierre SD, Desjardins D (2003) Wireless telephones and the risk of road crashes. Accid Anal Prev 35:649-660

62. Wilson J, Fang M, Wiggins S, Cooper P (2003) Collision and violation involvement of drivers who use cellular telephones. Traffic Inj Prev 4:45-52

63. Sullman M, Baas P (2004) Mobile phone use amongst New Zealand drivers. Transportation Research Part F: Traffic Psychology and Behaviour 7:95-105

64. McEvoy S, Stevenson M, McCartt A, Woodward M, Haworth C, et al (2005) Role of mobile phones in motor vehicle crashes resulting in hospital attendance: a case-crossover study. BMJ 331:428

65. Nabi H, Salmi R, Lafont S, Chiron M, Zins M, Lagarde E (2007) Attitudes associated with behavioral predictors of serious road traffic crashes: results from the GAZEL cohort. Injury Prevention 13:26-31 
66. Young RA, Schreiner C (2009) Real-world personal conversations using a hands-free embedded wireless device while driving: effect on airbag-deployment crash rates. Risk Anal 29:187-204

67. Greenland S (1982) The effect of misclassification in matched-pair case-control studies. Am J Epidemiol 116:402-406

68. Greenland S, Kleinbaum D (1983) Correcting for misclassification in two-way tables and matched-pair studies. Int J Epidemiology 12:93-97

69. Grender JM, Johnson WD (1993) Analysis of crossover designs with multivariate response. Stat Med 12:69-89

70. Marshall RJ, Jackson RT (1993) Analysis of case-crossover designs. Stat Med 12:23332341

71. Roberts I, Marshall R, Lee-Joe T (1995) The urban traffic environment and the risk of child pedestrian injury: a case-crossover approach. Epidemiology 6:169-171

72. Redelmeier D, Tibshirani R (2001) Car phones and car crashes: some popular misconceptions. CMAJ 164:1581-1582

73. Tibshirani R, Redelmeier D (1997) Cellular telephones and motor-vehicle collisions; some variations on matched-pairs analysis. The Canadian Journal of Statistics 25:581-591

74. Redelmeier D, Tibshirani R, Evans L (2003) Traffic-law enforcement and risk of death from motor-vehicle crashes: case-crossover study. Lancet 361:2177-2182

75. Laberge-Nadeau C, Bellavance F, Angers J, Maag U, et al (2006) Crash risk and cell phone use: Important questions on the real risk for legal decision makers. Valdor Conference, Stockolm, pp 305-314

76. Braver ER, Lund AK, McCartt AT (2009) Hands-free embedded cell phones and airbagdeployment crash rates. Risk Anal 29:1069, author reply 1070-1

77. Gibson JE, Hubbard RB, Smith CJ, Tata LJ, Britton JR, Fogarty AW (2009) Use of selfcontrolled analytical techniques to assess the association between use of prescription medications and the risk of motor vehicle crashes. Am J Epidemiol 169:761-768

78. Schouten H, Kester A (2009) A simple analysis of a simple crossover trial with a dichotomous outcome measure. Stat Med 29:193-198

79. Caird J, Scialfa C, Geoffrey H, Smiley A (2004) Effects of cellular telephones on driving behaviour and crash risk: results of meta-analysis. CAA Foundation for traffic safety, University of Calgary, 39p

80. McCartt A, Hellinga L, Bratiman K (2006) Cell phones and driving: review of research. Traffic Inj Prev 7:89-106

81. Brace C, Young K, Regan M (2007) Analysis of the literature: the use of mobiles phones while driving. Vagverket, Monash University, Accident Research Centre, 35p

82. INSPQ (2007) Avis de santé publique sur les effets du cellulaire au volant et recommandations. Institut National de santé publique du Québec, 85p

83. ONISR (2008) La sécurité routière en France, bilan de l'année 2007. La Documentation Française, Paris

84. Chapelon J, Sibi P (2007) Le téléphone portable au volant. Observatoire national interministériel de sécurité routière, Rapport 28 
85. Klauer S, Dingus V, Neale J, Sudweeks JD, Ramsey DJ (2006) The impact of driver inattention on near-crash/crash risk: an analysis using the 100 car naturalistic driving study data. NHTSA, National Technical Information Service, Virginia, 182p

86. Olson R, Hanowski R, Hickman J, Bocanera J (2009) Driver distraction in commercial vehicle operations. US DoT, Federal Motor Carrier Safety Administration, 49p

87. Guo F, Klauer S, Hankey J, Dingus T (2010) Near crashes as crash surrogate for naturalistic driving studies. Transportation Research Record 2147:66-74

88. Sayer J, Devonshire J, Flannagan C (2005) The effects of secondary tasks on naturalistic driving performance. UMTRI, University of Michigan, 48p

89. Hickman J, Hanowski R, Bocanegra J (2010) Distraction in commercial trucks and buses: Assessing prevalence and risk in conjunction with crashes and near-crashes. US Department of Transportation: p79

90. Shankar, V, P P Jovanis, et al (2008) Analysis of naturalistic ddriving data, prospective view on methodological paradigms. Transportation Research Record 2061:1-8

91. Jovanis, P P, V Shankar, et al (2010) Analysis of existing data: on methodological paradigms. Transportation Research Board of the National Academies Washington, DC, Larson Transportation Institute Pensylvania State University, University Park 